\title{
Serial absolute lactate value $<4$ versus relative $10 \%$ reduction as a predictor of mortality in severe sepsis and septic shock
}

\author{
S Lokhandwala ${ }^{1,2^{*}}$, P Patel $^{3}$, MN Cocchi ${ }^{2,3,4}$, MW Donnino $2,3,5$ \\ From ESICM LIVES 2015 \\ Berlin, Germany. 3-7 October 2015
}

\begin{abstract}
Introduction
Serial lactate measurements provide a valuable tool to risk-stratify patients, determine the presence of ongoing tissue hypoperfusion, and potentially evaluate efficacy of therapeutic interventions. Prior studies have shown lactate clearance to be a valuable predictor of patient outcomes, however some have defined adequate change in lactate by a decrease from baseline by $10 \%$ whereas others considered an absolute reduction to lactate $<4 \mathrm{mmol} / \mathrm{L}$. We hypothesize that a serial absolute lactate $<4 \mathrm{mmol} / \mathrm{L}$ after initial resuscitation will be as effective a predictor of future mortality as a change by $10 \%$.
\end{abstract}

\section{Objectives}

To compare the diagnostic characteristics of an absolute serial lactate $<4 \mathrm{mmol} / \mathrm{L}$ after resuscitation with the more traditional definition ( $>10 \%$ decrease in serum lactate).

\section{Methods}

Single-center retrospective study of patients presenting to an urban tertiary care Emergency Department (ED) with lactate $>4 \mathrm{mmol} / \mathrm{L}$ and suspected infection. Continuous data was analyzed using a one-way ANOVA, whereas categorical data was compared using Fisher's exact test. Patients were stratified by lactate clearance using the traditional definition ( $>10 \%$ decrease in serum lactate) as well as a novel definition (second lactate $<4 \mathrm{mmol} / \mathrm{L}$ ) and compared.

\section{Results}

Median initial lactate was $5.2 \mathrm{mmol} / \mathrm{L}$ [IQR 4.4-6.8]. The average decrease in serum lactate was $26.7 \%$ [2.3-44.4].
In-hospital mortality was $26.7 \% .109 / 161$ (67.7\%) patients had $>10 \%$ decrease in serum lactate, whereas $76 / 161$ $(47.2 \%)$ cleared lactate to $<4 \mathrm{mmol} / \mathrm{L}$. The average amount of crystalloid fluid resuscitation received was $2,970 \mathrm{ml}$, and the average time between lactate values was 181 minutes. Among patients who cleared lactate by at least $10 \%$, mortality was $21.1 \%$ while those who did not clear their lactate by $10 \%$ had $38.5 \%$ mortality $(\mathrm{p}=0.02)$. The negative predictive value for in-hospital mortality among those who clear their lactate by $10 \%$ was $78.9 \%$ [95\% CI 69.8-85.9], sensitivity was $46.5 \%$ [95\% CI: $31.5-62.2$ ] and specificity was $72.9 \%$ [95\% CI: 63.8-80.5]. Among patients who cleared their lactate to $<4 \mathrm{mmol} / \mathrm{L}$, mortality was $14.5 \%$, while those who did not clear their lactate to $<4 \mathrm{mmol} / \mathrm{L}$ had a mortality of $37.6 \%(\mathrm{p}=0.001)$. The negative predictive value for in-hospital mortality among those who clear lactate to $<4 \mathrm{mmol} / \mathrm{L}$ was $85.5 \%$ [ $95 \%$ CI 75.2-92.2], sensitivity was $74.4 \%$ [58.5-86.0] and specificity was $55.1 \%$ [45.7-64.2].

\section{Conclusions}

A serial lactate $<4 \mathrm{mmol} / \mathrm{L}$ as compared to $>10 \%$ change has improved negative predictive value for in-hospital mortality and may provide more utility for risk stratification, assessment of response to therapy, and potentially clinical decision-making.

\section{Grant Acknowledgment}

Dr. Donnino, the Primary Investigator, is supported by NHLBI 1K02HL107447-01A1 and Dr. Cocchi is supported by AHA 15SDG22420010. 
Table 1. Baseline Characteristics

\begin{tabular}{|c|c|c|c|c|c|}
\hline & $\begin{array}{c}\text { Lactate decrease }<10 \% \\
\mathrm{n}=52(32.3)\end{array}$ & $\begin{array}{c}\text { Lactate decrease }>10 \% \\
n=109(67.7)\end{array}$ & $\begin{array}{c}\text { Serial Lactate }>4 \mathrm{mmol} / \\
\mathrm{Ln}=85(52.8)\end{array}$ & $\begin{array}{c}\text { Serial Lactate }<4 \mathrm{mmol} / \\
\mathrm{L} n=76(47.2)\end{array}$ & Overall \\
\hline Age & $67.2+18.4$ & $69.3+15.2$ & $68.4+17.9$ & $69.0+14.4$ & $\begin{array}{c}68.6 \\
+16.3\end{array}$ \\
\hline Initial Lactate, $\mathrm{mmol} / \mathrm{L}$ & $5.25[4.4-7.1]$ & $5.2[4.4-6.7]$ & $6[4.9-8.6]$ & $4.7[4.3-5.3]$ & $\begin{array}{l}5.2[4.4- \\
6.8]\end{array}$ \\
\hline$\%$ Decrease in Lactate & $0.0[-13.3-2.26]$ & $37.3[25.6-52.7]$ & $3.9[-3.7-21.0]$ & $44.4[32.4-58.1]$ & $\begin{array}{c}26.7[2.3- \\
44.4]\end{array}$ \\
\hline $\begin{array}{l}\text { Time between lactate } \\
\text { values, minutes }\end{array}$ & $168+109.7$ & $188+80.2$ & $163+99.5$ & $202+75.9$ & $\begin{array}{c}181 \\
+90.9\end{array}$ \\
\hline $\begin{array}{l}\text { Volume Crystalloid in } 3 \\
\text { hours, } \mathrm{ml}\end{array}$ & $2971+1931$ & $2969+1499$ & $3080+1865$ & $2850+1358$ & $\begin{array}{c}2970 \\
+1640 \\
\end{array}$ \\
\hline $\begin{array}{l}\text { Initial Systolic Blood } \\
\text { Pressure }\end{array}$ & $105+31.0$ & $100+23.6$ & $105.9+28.7$ & $97.8+22.7$ & $\begin{array}{l}102.0 \\
+26.2\end{array}$ \\
\hline $\begin{array}{l}\text { Vasopressors Use within } \\
3 \text { hours }\end{array}$ & $22(42.3)$ & $32(29.4)$ & $35(41.2)$ & $19(25.0)$ & $54(33.5)$ \\
\hline ICU LOS, days & $2.1[1.1-4.6]$ & $2.2[1.3-5.9]$ & $2.7[1.2-5.9]$ & $1.9[1.1-5.4]$ & $\begin{array}{c}2.2[1.1- \\
5.7]\end{array}$ \\
\hline In-Hospital Mortality & $20(38.5)$ & $23(21.1)$ & $32(37.6)$ & $11(14.5)$ & $43(26.7)$ \\
\hline
\end{tabular}

Table 2. Comparison based on Lactate Clearance

\begin{tabular}{|c|c|c|c|c|c|c|}
\hline & $\begin{array}{c}\text { Lactate decrease } \\
<10 \% \\
\end{array}$ & $\begin{array}{c}\text { Lactate decrease }> \\
10 \% \\
\end{array}$ & $\begin{array}{c}\mathrm{P}- \\
\text { value }\end{array}$ & $\begin{array}{c}\text { Serial Lactate > } \\
4 \mathrm{mmol} / \mathrm{L}\end{array}$ & $\begin{array}{c}\text { Serial Lactate } \\
<4 \mathrm{mmol} / \mathrm{L}\end{array}$ & P-value \\
\hline $\mathrm{N}$ & 52 & 109 & - & 85 & 76 & - \\
\hline Initial Lactate, $\mathrm{mmol} / \mathrm{L}$ & 6.1 & 5.9 & 0.43 & 6.8 & 4.9 & $\begin{array}{c}< \\
0.0001 \\
\end{array}$ \\
\hline $\begin{array}{l}\text { Volume Crystalloid in } 3 \\
\text { hours, } \mathrm{ml}\end{array}$ & 2971.7 & 2969.4 & 0.99 & 2850.5 & 3080.6 & 0.39 \\
\hline Vasopressors within 3 hours & $22(42.3)$ & $32(29.4)$ & 0.11 & $35(41.2)$ & $19(25.0)$ & 0.04 \\
\hline In-hospital Mortality & $20(38.5)$ & $23(21.1)$ & 0.02 & $32(37.7)$ & $11(14.5)$ & 0.001 \\
\hline
\end{tabular}

\section{Authors' details}

'Beth Israel Deaconess Medical Center, Internal Medicine, Boston, United States. ${ }^{2}$ Harvard Medical School, Boston, United States. ${ }^{3}$ Beth Israel Deaconess Medical Center, Emergency Medicine, Boston, United States. ${ }^{4}$ Beth Israel Deaconess Medical Center, Anesthesia Critical Care, Boston, United States. ${ }^{5}$ Beth Israel Deaconess Medical Center, Pulmonary and Critical Care, Boston, United States.

Published: 1 October 2015

doi:10.1186/2197-425X-3-S1-A359

Cite this article as: Lokhandwala et al: Serial absolute lactate value $<4$ versus relative $10 \%$ reduction as a predictor of mortality in severe sepsis and septic shock. Intensive Care Medicine Experimental 2015 3(Suppl 1):A359.

\section{Submit your manuscript to a SpringerOpen ${ }^{\circ}$ journal and benefit from:}

- Convenient online submission

- Rigorous peer review

- Immediate publication on acceptance

- Open access: articles freely available online

- High visibility within the field

- Retaining the copyright to your article

Submit your next manuscript at $>$ springeropen.com 